\title{
COMMENTARY
}

\section{Septic acute kidney injury: hemodynamic syndrome, inflammatory disorder, or both?}

\author{
Miklos Lipcsey ${ }^{1,2}$ and Rinaldo Bellomo ${ }^{3 *}$ \\ For related research see Benes et al., http://ccforum.com/content/15/5/R256
}

\begin{abstract}
Septic acute kidney injury (S-AKI) is the most common cause of kidney injury in the ICU. Decreased renal blood flow and inflammation have both been suggested as mechanisms of S-AKI. Benes and colleagues present a study of S-AKI in which sepsis is induced by fecal peritonitis and bacterial infusion. In this study, although decreased renal blood flow and increased renal vascular resistance were present in some of the animals that developed S-AKI, inflammatory activation without decreased renal blood flow and increased renal vascular resistance was seen in other animals. Systemic hemodynamic findings provided little information on renal hemodynamics or risk of S-AKI. The study highlights the extraordinary complexity of S-AKI and the need for clinicians to recognize our limited understanding of its pathogenesis and the weakness of the decreased perfusion paradigm as the sole explanation for the loss of renal function seen in severe sepsis.
\end{abstract}

\section{We know accurately only when we know little; with knowledge doubt increases.}

Goethe

The pathophysiology of septic acute kidney injury (S-AKI) is poorly understood $[1,2]$, yet $\mathrm{S}-\mathrm{AKI}$ is a major complication of sepsis and sepsis is the most common cause of acute kidney injury in the ICU [3]. The conventional explanation for the development of S-AKI emphasizes the role of hypoperfusion [4]. Despite the dominance of such views, however, the evidence to

\footnotetext{
*Correspondence: rinaldo.bellomo@austin.org.au

${ }^{3}$ Australian and New Zealand Intensive Care Research Centre, School of Public Health and Preventive Medicine, Monash University, Alfred Centre, Commercial Road, Prahran, Melbourne, Victoria 3181, Australia

Full list of author information is available at the end of the article
}

support them is weak. This is especially true because renal perfusion (renal blood flow (RBF)) cannot be reliably measured in man. Importantly, changes in intraRBF distribution and glomerular hemodynamics may account for loss of function, even in the presence of maintained or increased RBF [5,6]. In addition, conventional views of the pathophysiology of S-AKI have been derived from models of acute kidney injury with limited relevance to human disease and, when different models are studied, they deliver different changes in RBF and function [6]. Finally, the role of nitric oxide [7] and the role of nonhemodynamic mechanisms such as apoptosis and the innate immune response remain poorly understood. It is in this context that one should assess the experiments from Matejovic's group [1].

Using two porcine models of sepsis, the investigators studied some pathophysiological phenomena associated with S-AKI. Sepsis was induced either by peritonitis, with peritoneal autologous feces inoculation, or by continuous intravenous infusion of live bacteria. The animals were resuscitated as needed and observed for 22 hours, simulating the clinical situation. Monitoring of global and local circulation, inflammatory and oxidative stress markers was performed. S-AKI developed in $62 \%$ of among animals with peritonitis, compared with $40 \%$ in the bacterial infusion group, despite the fact that the peritonitis group had a significantly greater systemic oxygen delivery. S-AKI animals had greater inflammatory and oxidative stress and demonstrated a diverging late renal circulatory pattern of higher renal vascular resistance, decreased RBF, and earlier decrease in renal cortical microcirculatory blood flow.

These findings suggest that S-AKI could be induced by both renal circulatory alterations and inflammatory injury, with the large variability in the measured parameters implying that either of these mechanisms could dominate in a given subject. Some of the renal circulatory alterations suggest that S-AKI may have a local ischemic component. However, these changes occurred late in the experiment and were preceded by deterioration of renal functional markers, and thus cannot fully explain the development of S-AKI. 
Furthermore, renal vascular resistance was more than five times greater in the bacterial infusion animals that developed S-AKI than in the peritonitis animals that also developed S-AKI, suggesting that increased renal vascular resistance is not necessary for the development of S-AKI. This provocative observation raises the possibility that S-AKI can occur within the setting of at least two different renal hemodynamic phenotypes: vasodilatory or vasoconstrictive, depending on the type of infection. Finally, a striking feature of these data is the lack of difference in systemic circulatory variables between S-AKI and non-S-AKI animals, suggesting that the systemic hemodynamic phenotype provides little guide to predicting the development of S-AKI or the renal hemodynamic subtype of S-AKI.

The above observations support the notion that, although S-AKI can be a consequence of renal ischemia under some circumstances, this is not a necessary feature. In fact, increased RBF is not unusual in S-AKI models [6], and neither renal medullary nor cortical blood flow is predictably altered [8]. The uncoupling of RBF and glomerular filtration can have other explanations. Efferent arteriolar vasodilatation may increase RBF but decrease glomerular filtration pressure and urine production [9]. Perhaps, even more provocatively, the organization of the glomerular circulation may be far more complex than previously anticipated [10] and may permit direct shunting between the afferent and efferent arterioles (periglomerular circulation). Efferent arteriolar vasodilatation, periglomerular shunting, blood flow redistribution within the kidney and, in some cases, late vasoconstriction can thus all participate in the pathogenesis of S-AKI at different times and in different situations.

As Benes and colleagues describe, however, some of the pathophysiology behind S-AKI may be inflammatory in nature. In fact, it is known that TNF $\alpha$ can induce acute kidney injury [11]. Benes and colleagues found an early increase in TNF $\alpha$ in animals developing S-AKI. More recent work additionally highlights the role that bactericidal antibiotic-induced release of cytokine alone can play in triggering S-AKI [12]. Other substances with a dual role (markers and mediators of tissue damage) are reactive oxygen species causing oxidative stress. Such markers increased early and were highest in pigs with peritonitisinduced S-AKI. It remains uncertain, however, to what extent cytokines and oxygen radicals are biomarkers, true mediators of injury, or both under these circumstances.

Both renal hemodynamic changes and inflammatory activation are thus major phenomena in S-AKI as supported by Benes and colleagues' study. The slow evolution of sepsis, the clinically relevant resuscitation and the long observation period greatly increase the relevance of their study. Lack of data for the first 12 hours of the experiment, when a porcine hypodynamic circulation is not unusual and could contribute to S-AKI development, creates some uncertainty about hemodynamics; and the lack of tubular biomarkers of injury [13] limits our understanding of the effects of these models on tubular cells. Yet, the insights provided by comparing two sepsis models and by simultaneously extensively measuring circulatory and inflammatory variables are clearly valuable. These insights add much-needed data to this field and highlight how much, as knowledge increases, doubt increases further.

\section{Abbreviations}

RBF, renal blood flow; S-AKI, septic acute kidney injury; TNF, tumor necrosis factor.

\section{Competing interests}

The authors declare that they have no competing interests.

\section{Author details}

'Department of Intensive Care, Austin Hospital, 145 Studley Road, Heidelberg, Melbourne 3084, Australia. ${ }^{2}$ Department of Surgical Sciences, Anaesthesiology and Intensive Care, Uppsala University Hospital, Akademiska sjukhuset, 751 85 Uppsala, Sweden. ${ }^{3}$ Australian and New Zealand Intensive Care Research Centre, School of Public Health and Preventive Medicine, Monash University, Alfred Centre, Commercial Road, Prahran, Melbourne, Victoria 3181, Australia.

Published: 18 November 2011

\section{References}

1. Benes J, Chvojka J, Sykora R, Radej J, Krouzecky A, Novak I, Matejovic M: Searching for mechanisms that matter in early septic acute kidney injury: an experimental study. Crit Care 2011, 15:R256.

2. Ricci Z, Ronco C: Pathogenesis of acute kidney injury during sepsis. Curr Drug Targets 2009, 10:1179-1183.

3. Uchino S, Kellum JA, Bellomo R, Doig GS, Morimatsu H, Morgera S, Schetz M, Tan I, Bouman C, Macedo E, Gibney N, Tolwani A, Ronco C: Acute renal failure in critically ill patients: a multinational, multicenter study. JAMA 2005, 294:813-818.

4. Schrier RW, Wang W: Acute renal failure and sepsis. N Engl J Med 2004, 351:159-169.

5. Brenner M, Schaer GL, Mallory DL, Suffredini AF, Parrillo JE: Detection of renal blood flow abnormalities in septic and critically ill patients using a newly designed indwelling thermodilution renal vein catheter. Chest 1990, 98:170-179.

6. Langenberg C, Bellomo R, May C, Wan L, Egi M, Morgera S: Renal blood flow in sepsis. Crit Care 2005, 9:R363-R374.

7. Ishikawa K, Bellomo R, May CN: The impact of intrarenal nitric oxide synthase inhibition on renal blood flow and function in mild and severe hyperdynamic sepsis. Crit Care Med 2011, 39:770-776.

8. Di Giantomasso D, Morimatsu H, May CN, Bellomo R: Intrarenal blood flow distribution in hyperdynamic septic shock: effect of norepinephrine. Crit Care Med 2003, 31:2509-2513.

9. Wan L, Langenberg C, Bellomo R, May CN: Angiotensin II in experimental hyperdynamic sepsis. Crit Care 2009, 13:R190.

10. Molitoris BA, Sandoval RM: Kidney endothelial dysfunction: ischemia, localized infections and sepsis. In Controversies in Acute Kidney Injury. Edited by Kellum JA, Ronco C, Vincent JL. Basel: Karger; 2011:108-118.

11. Tracey KJ, Beutler B, Lowry SF, Merryweather J, Wolpe S, Milsark IW, Hariri RJ, Fahey TJ, 3rd, Zentella A, Albert JD, Shires GT, Cerami A: Shock and tissue injury induced by recombinant human cachectin. Science 1986, 234:470-474

12. Peng ZY, Wang HZ, Srisawat N, Wen X, Rimmele T, Bishop J, Singbartl K, Murugan R, Kellum JA: Bactericidal antibiotics temporarily increase inflammation and worsen acute kidney injury in experimental sepsis. Crit Care Med 2011. [Epub ahead of print]

13. Bagshaw SM: Subclinical acute kidney injury: a novel biomarker-defined syndrome. Crit Care Resusc 2011, 13:201-203.

\section{doi:10.1186/cc10525}

Cite this article as: Lipcsey M, Bellomo R: Septic acute kidney injury: hemodynamic syndrome, inflammatory disorder, or both? Critical Care 2011, 15:1008. 